\title{
Evidence for a common progenitor of epithelial and mesenchymal components of the liver
}

\author{
A Conigliaro ${ }^{1,5}$, L Amicone ${ }^{1,5}$, V Costa ${ }^{2}$, M De Santis Puzzonia ${ }^{1}$, C Mancone ${ }^{2}$, B Sacchetti ${ }^{3}$, C Cicchini ${ }^{1}$, F Garibaldi ${ }^{1}$, \\ DA Brenner ${ }^{4}$, T Kisseleva ${ }^{4}$, P Bianco ${ }^{3}$ and M Tripodi ${ }^{*, 1,2}$
}

Tissues of the adult organism maintain the homeostasis and respond to injury by means of progenitor/stem cell compartments capable to give rise to appropriate progeny. In organs composed by histotypes of different embryological origins (e.g. the liver), the tissue turnover may in theory involve different stem/precursor cells able to respond coordinately to physiological or pathological stimuli. In the liver, a progenitor cell compartment, giving rise to hepatocytes and cholangiocytes, can be activated by chronic injury inhibiting hepatocyte proliferation. The precursor compartment guaranteeing turnover of hepatic stellate cells (HSCs) (perisinusoidal cells implicated with the origin of the liver fibrosis) in adult organ is yet unveiled. We show here that epithelial and mesenchymal liver cells (hepatocytes and HSCs) may arise from a common progenitor. Sca + murine progenitor cells were found to coexpress markers of epithelial and mesenchymal lineages and to give rise, within few generations, to cells that segregate the lineage-specific markers into two distinct subpopulations. Notably, these progenitor cells, clonally derived, when transplanted in healthy livers, were found to generate epithelial and mesenchymal liver-specific derivatives (i.e. hepatocytes and HSCs) properly integrated in the liver architecture. These evidences suggest the existence of a 'bona fide' organ-specific meso-endodermal precursor cell, thus profoundly modifying current models of adult progenitor commitment believed, so far, to be lineage-restricted. Heterotopic transplantations, which confirm the dual differentiation potentiality of those cells, indicates as tissue local cues are necessary to drive a full hepatic differentiation. These data provide first evidences for an adult stem/ precursor cell capable to differentiate in both parenchymal and non-parenchymal organ-specific components and candidate the liver as the instructive site for the reservoir compartment of HSC precursors as yet non-localized in the adult.

Cell Death and Differentiation (2013) 20, 1116-1123; doi:10.1038/cdd.2013.49; published online 17 May 2013

In many postnatal organs, physiological cell turnover and restoration of cell loss after injury are maintained by progenitor/ stem cell compartments residing in specialized niches. ${ }^{1-3}$ Concerning the liver, its ability to regenerate is largely based on the proliferation of terminally differentiated parenchymal liver cells (hepatocytes and cholangiocytes); however, when the proliferation of mature epithelial cells is inhibited by exposure to drugs or by chronic injury, a progenitor cell compartment emerges. ${ }^{4,5}$ These progenitor cells, known as 'oval cells' in rodents, and proven to constitute a heterogeneous cell population, ${ }^{6}$ are characterized by the expression of markers of both cholangiocytes and hepatocytes and by a high proliferative potential in all species. The progenitors are regarded as bipotential transient amplifying cells, ${ }^{7,8}$ downstream of a normally quiescent true stem cell. With respect to the mesenchymal hepatic stellate cells (HSC), while their embryonic derivation from septum transversum mesenchyme and from mesothelium has been demonstrated with elegant experiments of cell lineage tracing, ${ }^{9}$ their progenitor compartment in the adult liver has not been identified.
HSCs, liver resident cells occupying the space of Disse between hepatocytes and sinusoids, ${ }^{10-12}$ have a unique and critical role in liver physiology and pathology, as they regulate several functions including storage and release of retinoids and presentation of lipid antigens to NK cells; ${ }^{13}$ moreover, in tissue response to chronic injury, they proliferate and convert into fibrogenic myofibroblasts leading to fibrosis. ${ }^{14}$

We previously isolated from murine liver explants and stabilized in several lines a Sca1 + CD34-, CD45-, albuminprogenitor cell (RLSC, from resident liver stem cell) capable to differentiate spontaneously (i.e. without instructive culture conditions) into hepatocytes. ${ }^{15,16}$ In this study, we show the characterization of three different cell lines derived by cloning procedures from three independent RLSC lines: these cells coexpress stem, epithelial and mesenchymal markers and are able to differentiate, in vitro, in subpopulations expressing either mesenchymal or epithelial genes. Heterotopic and orthotopic transplants have been performed in order to characterize the in vivo differentiation capacity of RLSCs. Surprisingly, RLSCs transplanted in the growing

\footnotetext{
${ }^{1}$ Istituto Pasteur-Fondazione Cenci Bolognetti, Department of Cellular Biotechnologies and Haematology, Sapienza University of Rome, Rome, Italy; ${ }^{2}$ L. Spallanzani National Institute for Infectious Diseases, IRCCS, Rome, Italy; ${ }^{3}$ Department of Molecular Medicine, Sapienza University of Rome, Rome, Italy and ${ }^{4}$ Department of Medicine, University of California, San Diego, La Jolla, CA, USA

*Corresponding author: M Tripodi, Dipartimento di Biotecnologie Cellulari ed Ematologia, Sezione di Genetica, Molecolare, Sapienza University of Rome, Viale Regina Elena 324, 00161 Rome, Italy. Tel: +39 06 4461387; Fax: +39 06 49918244; E-mail: tripodi@bce.uniroma1.it

${ }^{5}$ These authors contributed equally to this work.

Keywords: liver stem cell; hepatic stellate cell; cell transplantation; differentiation

Abbreviations: $\alpha \mathrm{FP}$, alpha fetoprotein; $\alpha \mathrm{SMA}$, alpha smooth muscle actin; CK, cytokeratin; EGFP, enhanced green fluorescent protein; EpCAM, epithelial cell adhesion molecule; GFAP, glial fibrillary acidic protein; HNF4 $\alpha$, hepatocyte nuclear factor 4 alpha; HSCs, hepatic stellate cells; RLSC, resident liver stem cell Received 31.10.12; revised 22.3.13; accepted 08.4.13; Edited by G Cossu; published online 17.5.13
} 
livers of newborn mice, contribute to both mesenchymal and epithelial hepatic lineages: they in fact differentiate into hepatocytes and mesenchymal cells that, for their subendothelial localization together with a GFAP (glial fibrillary acidic protein) and Desmin positivity, can be reconducted to HSCs.

\section{Results}

RLSCs co-express epithelial and mesenchymal markers and undergo into mutually exclusive differentiations. Three RLSC lines, isolated from embryonic livers at 14 days post coitum (E14/1-1 and E14/3-1) and from liver of a 6-daysold mouse (NB-D6) and previously characterized for their broad differentiation capacity, ${ }^{15}$ have been marked with a enhanced green fluorescent protein (EGFP)-expressing retrovirus, seeded at clonal limiting dilution ( 0.2 cell/well) and expanded. A single EGFP + clonal progeny from each of the starting lines was randomly selected. The results obtained in cell culture and in vivo experiments and described below were equivalent for the three clones.

FACS analysis of the undifferentiated RLSC clones revealed as the cells homogenously express the stem marker SCA1 and notably, coexpress epithelial and mesenchymal markers such as E-Cadherin, Pan-CK and Vimentin (Figure 1a). An immunocytochemical analysis confirmed the coexpression of these proteins (Figure 1b). A broader characterization by FACS analysis showed that cells were negative for CD144, CD45, CD90 and Thy1 (data not shown).

Surprisingly, low-serum culture condition gave rise, within the same culture, to cells with either epithelial or mesenchymal morphology (Figure 1c). The unexpected appearance of two morphologically discrete subpopulations within a clonal cell line rendered mandatory to gather observations at single cell level. To this end, we followed single cell proliferation/differentiation by live imaging. This analysis allowed to reconduct the dimorphism to single mother cells: as shown in Figures 1d and e and in the Supplementary Video, single EGFP-RLSC followed for 5 days gave rise to cells either coexpressing E-Cadherin and Vimentin or expressing one of these two markers in a mutually exclusive fashion.

To further characterize the segregation of epithelial and mesenchymal markers to different cell populations, a dimorphic cell culture obtained at confluence after 10 days of starvation was analysed by immunofluorescence. Staining for Vimentin/E-Cadherin highlighted as cells express these markers in a mutually exclusive fashion and in coherence with the corresponding cellular habit (rare double positive cells are still detectable). A further analysis for epithelial and mesenchymal markers (i.e. HNF $4 \alpha$ and $\alpha$ SMA), not expressed in the starting population, was performed. In line with the previous observation the epithelial-shaped progeny was found to acquire the HNF4 $\alpha$ expression, while the mesenchymalshaped cells were found positive for the myofibroblastassociate marker $\alpha$-smooth muscle actin ( $\alpha \mathrm{SMA})$; notably, as expected for lineage-specific markers not present in the starting population and acquired during differentiation, no double positive (HNF4 $\alpha / \alpha \mathrm{SMA}$ ) cells were observed
(Figure 2a). Quantitative analysis of markers segregation is shown in Figure 2b.

These results indicate an intrinsic dual differentiation potentiality of RLSCs.

RLSC derivatives display liver-specific epithelial and mesenchymal phenotypes in orthotopic transplant. To challenge in vivo the intrinsic dual differentiation potentiality of RLSC, EGFP + cells were injected directly into the liver of untreated immuno-tollerant (CD1-nude) mice at the first day after birth, through a percutaneous trans-abdominal puncture, according to Bissig et al. ${ }^{17}$ Animals were killed at various times and the peak of colonization was found around 2 months after cell transplantation. RLSC progeny, only capitalizing on the differentiating and proliferative stimuli of the growing organ and in competition with endogenous cells, was found to reproducibly colonize the recipient livers in 40/50 injected animals. In 30 animals, EGFP + cells expressing hepatocyte markers (i.e. the transcription factor HNF4 $\alpha$ and Albumin) engrafted in the hepatocytic muralium with efficiency until $0.01 \%$ of total hepatocytes (Figure 3 ). Notably, in each of the 40 engrafted mice, EGFP + cells with a mesenchymal shape and expressing GFAP and Desmin were also observed (Figure 4a). The subendothelial localization of these cells (in perisinusoidal spaces, Figure $4 b$ ) together with their immuno-phenotype correspond to those of HSCs.

Taken together, these data indicated that clonal RLSCs could contribute to both epithelial and mesenchymal specific compartments of the liver.

RLSCs give rise to liver-specific epithelial and mesenchymal derivatives in heterotopic transplants. In order to determine to what extent the differentiation of transplanted progenitors into hepatic lineages was induced by liverspecific local cues, RLSCs were transplanted subcutaneously into the back of SCID beige mice, as described in Materials and Methods. The cotransplants with HUVEC cells were performed in order to guarantee a homogenous vascularization inside the implant. Histological and immunohistochemical analysis of implants showed RLSC derivatives with both epithelial and mesenchymal phenotypes (Figure 5a). Although cells bearing an epithelial morphology, often organized in large ducts mimicking a glandular organization, were found to express a number of liver histotype-restricted markers including EpCAM, $\alpha$-fetoprotein, E-Cadherin, CK7, Albumin, those displaying a mesenchymal shape were found to express GFAP and $\alpha$ SMA (Figures $5 b$ and $\mathrm{c}$ ). The expression of EpCAM and $\alpha$-fetoprotein (endodermal precursor markers), in particular, suggests that in an extra-hepatic context, RLSC derivatives proliferate retaining precursor features; moreover, the low expression of Albumin and CK7 indicates also the RLSCs aptitude to differentiate toward hepatocytes/cholangiocytes. These data are extended by RT-PCR and western analysis as shown in Supplementary Figure 1 (see also Supplementary Materials and Table 1). With respect to mesenchymal differentiation, the expression of $\alpha$ SMA and GFAP suggested that the ability of RLSCs to generate an HSClike phenotype was also inherent and independent of exogenous, liver-restricted cues. Overall, heterotopic 

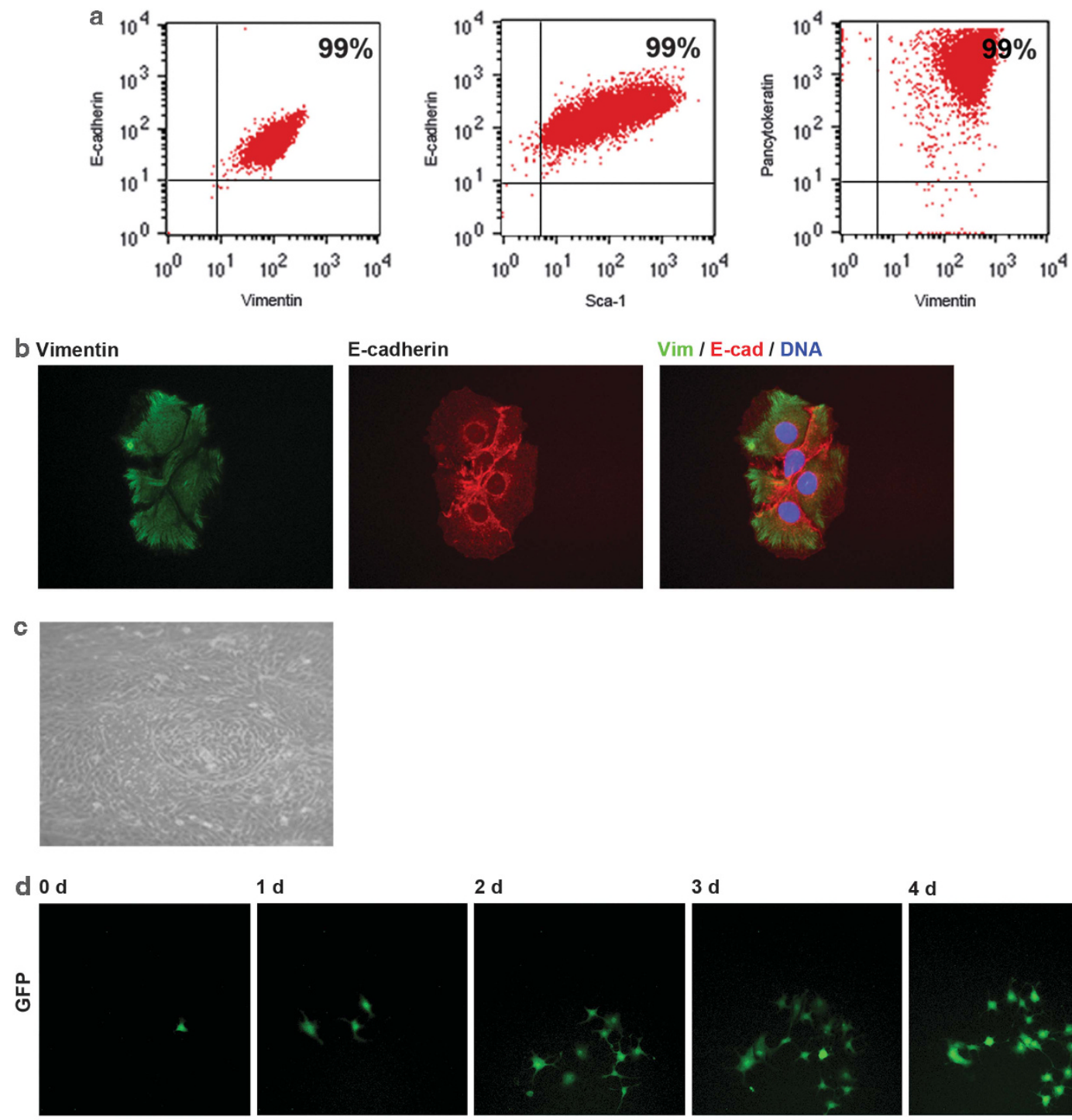

$1 \mathrm{~d}$

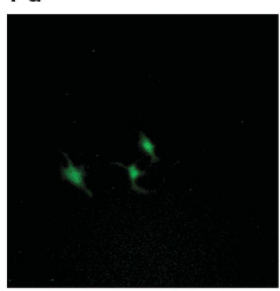

$2 \mathrm{~d}$

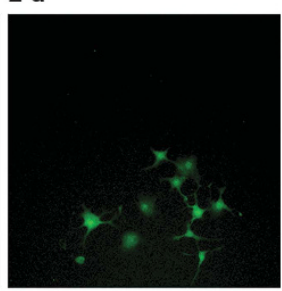

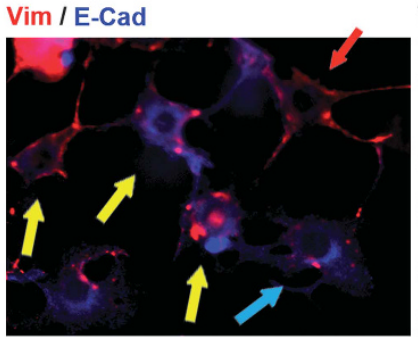

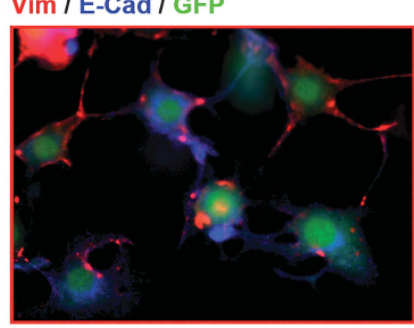

$3 \mathrm{~d}$

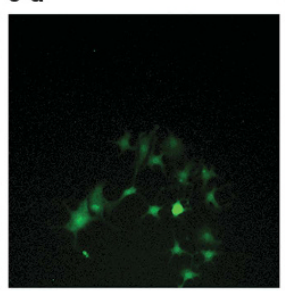

Vimentin

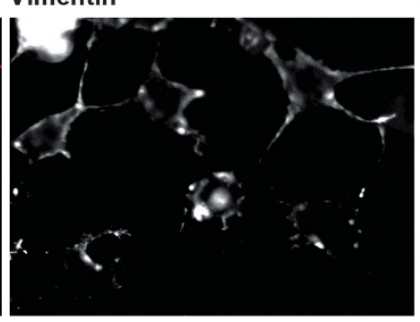

$4 \mathrm{~d}$

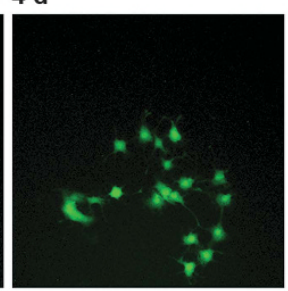

$5 \mathrm{~d}$

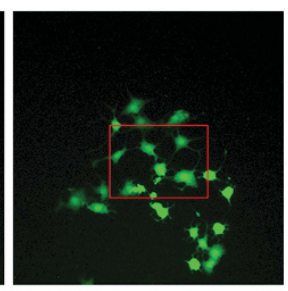

E-cadherin

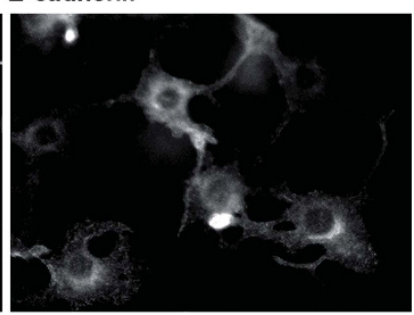

Figure 1 RLSC characterization and in vitro differentiation. (a) FACS analysis for the indicated antigens highlighted as the RLSC starting population homogeneously expresses the stemness marker SCA1, the epithelial markers Pan-CK and E-cad and the mesenchymal marker Vimentin. (b) Immunocytochemical analysis for the indicated markers highlighted as the RLSC starting population coexpresses epithelial and mesenchymal markers. (c) Within 10 days, RLSCs in low-serum culture condition gave rise to derivatives that acquired distinct epithelial and mesenchymal phenotypes, as observed by phase contrast (original magnification $\times 20$ ). (d-e) Time-lapse analysis (see also video 1). Within 5 days single EGFP-RLSC cells, in low-serum culture condition, gave rise to clonal progeny composed of cells coexpressing Vimentin and E-cadherin (yellow arrows) and cells expressing E-cadherin (blue arrow) or Vimentin (red arrow)

transplantations highlighted that RLSCs retain a binary, mesenchymal-epithelial potential, independent of soluble factors, cell-to-cell and cell-to-ECM organ-specific instructive signals. Taken together these results indicate an intrinsic dual potential of RLSCs to differentiate into either epithelial or mesenchymal derivatives. This dual potential, per se cell-autonomous as reproduced in cell culture, may harness organ-specific cues in vivo, critical for maturation toward liver-specific cell types and acquisition of proper tissue architecture. 
a

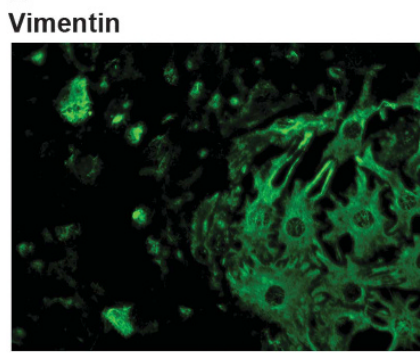

E-cadherin

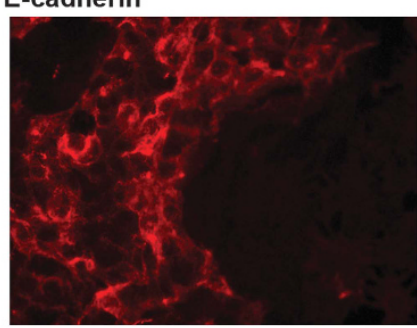

$\alpha$ SMA / DNA

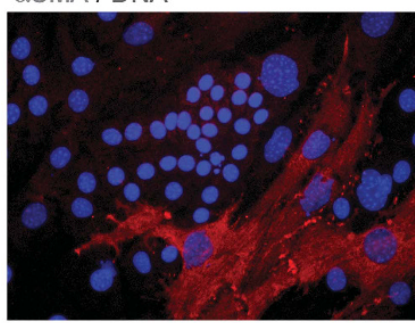

HNF4

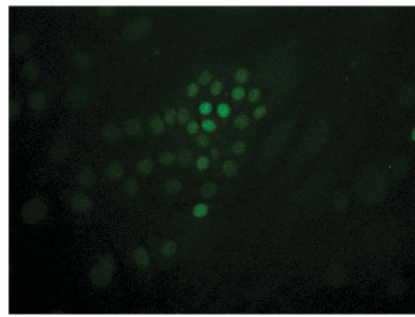

b

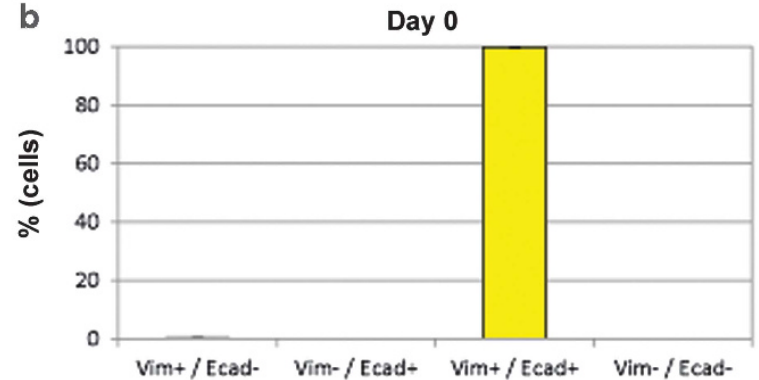

Vim / E-cad / DNA

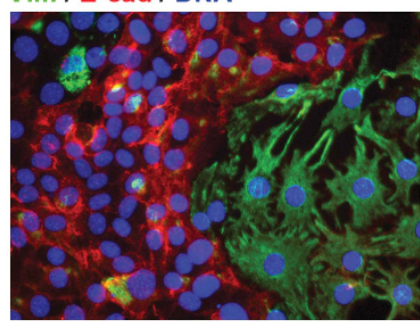

HNF4 / $\alpha$ SMA

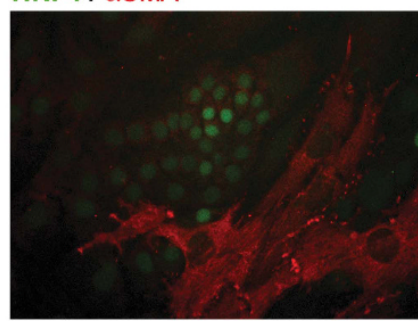

Phase Contrast

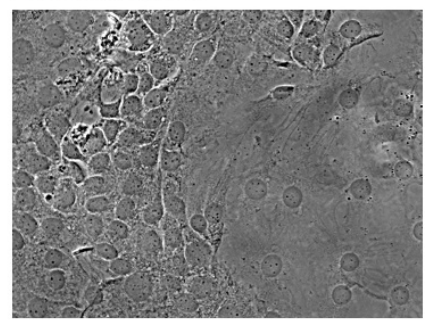

Phase Contrast

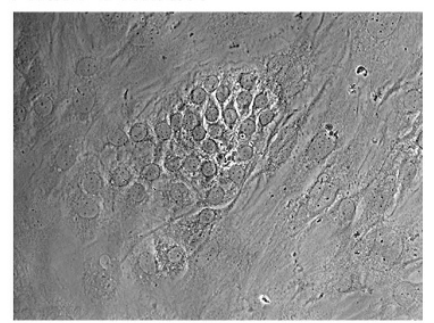

Day 10

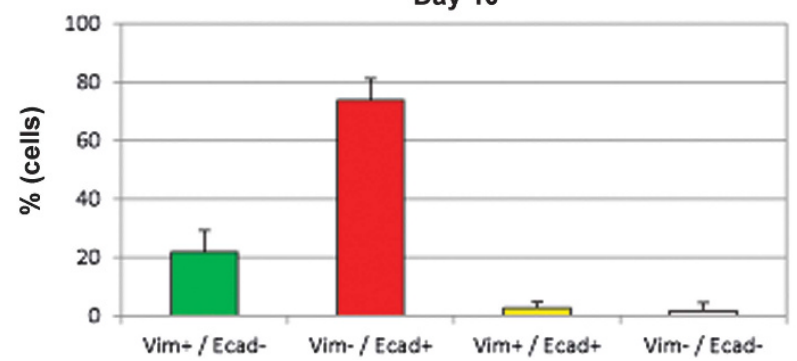

Figure 2 RLSC derivatives segregation of epithelial and mesenchymal markers into distinct subpopulations. (a) Immunocytochemical analysis for the indicated markers highlighted as the RLSCs, in low-serum culture condition for 10 days, undergo into either epithelial or mesenchymal differentiation. The morphology of the two different cellular subpopulations is coherent with the expression of specific markers. (b) Cells were scored in four categories based on their Vimentin and E-Cadherin expression. Data show percentage of cells for each category. Average of three experiments. $N=500$ cells were counted for each experiment. Data are shown as mean \pm S.E.M.

\section{Parenchymal cells}

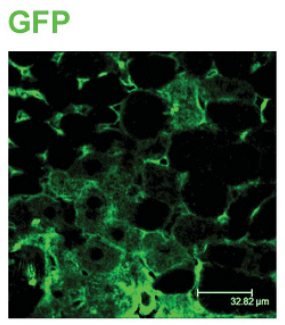

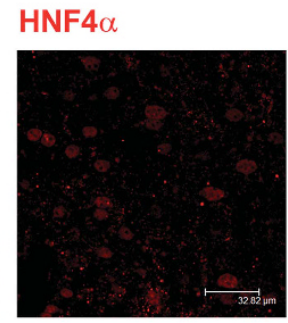

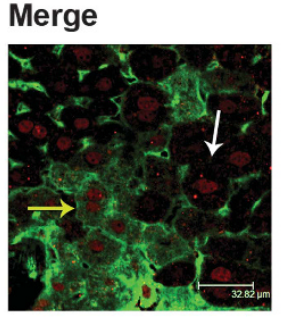

GFP
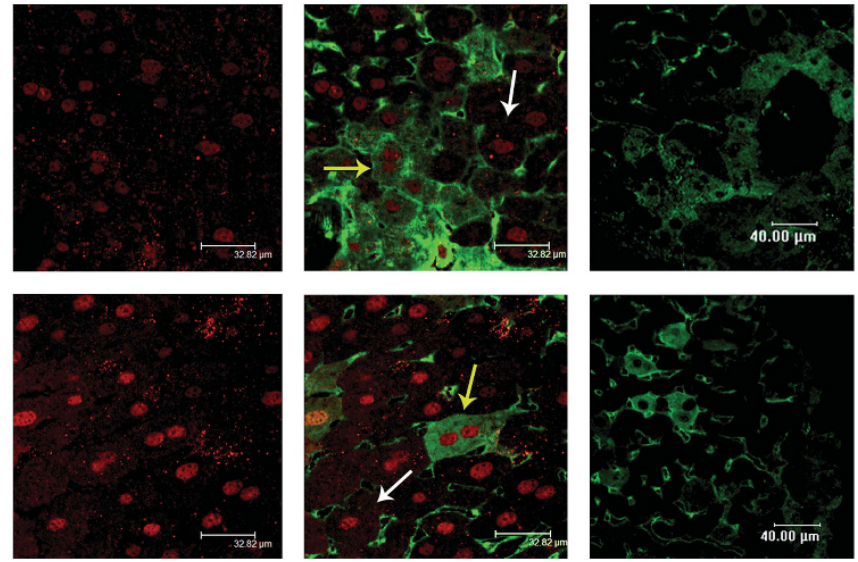
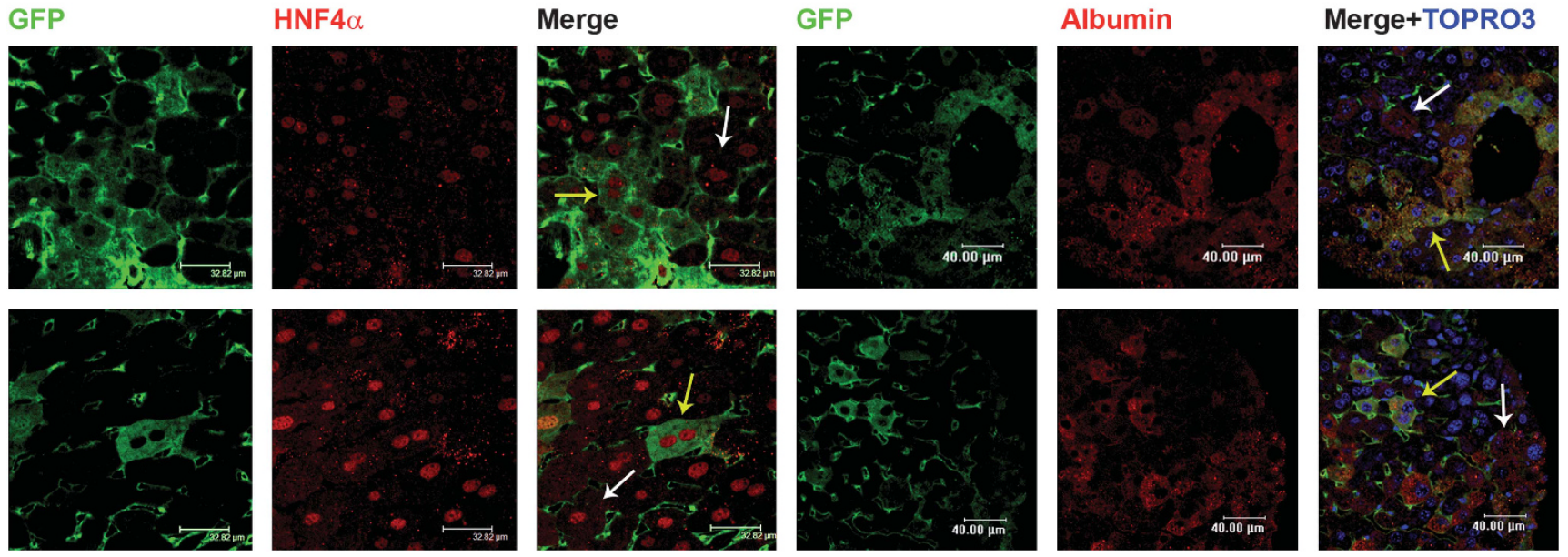

Figure 3 RLSC parenchymal derivatives in orthotopic transplants. Immunohistochemical analysis of RLSC-engrafted livers. The RLSC EGFP progeny was detected in untreated CD1 null mice injected at day 1 after birth and killed after 2 months. EGFP parenchymal cells were found integrated into the epithelial cords expressing hepatocyte markers HNF4 and Albumin. Yellow arrows indicate exogenous hepatocytes; white arrows indicate endogenous cells 
a
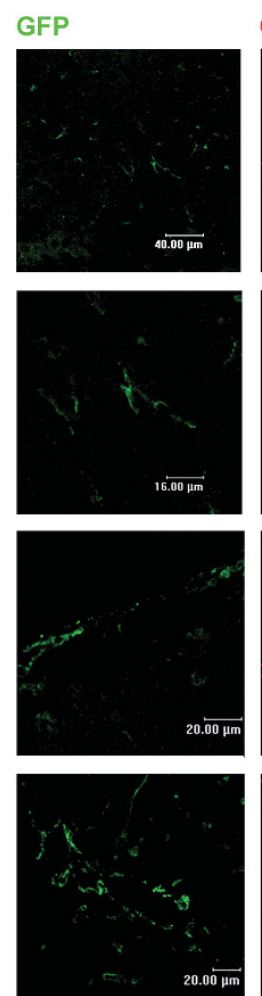

GFAP
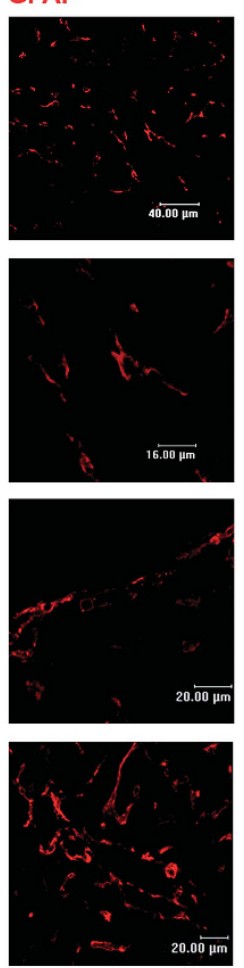

Non parenchymal cells
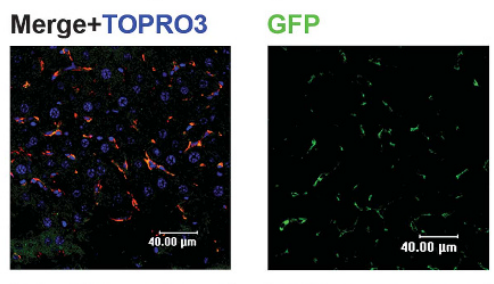

Desmin

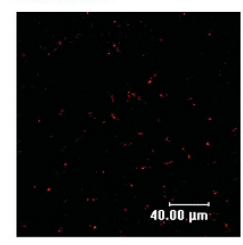

Merge+TOPRO3
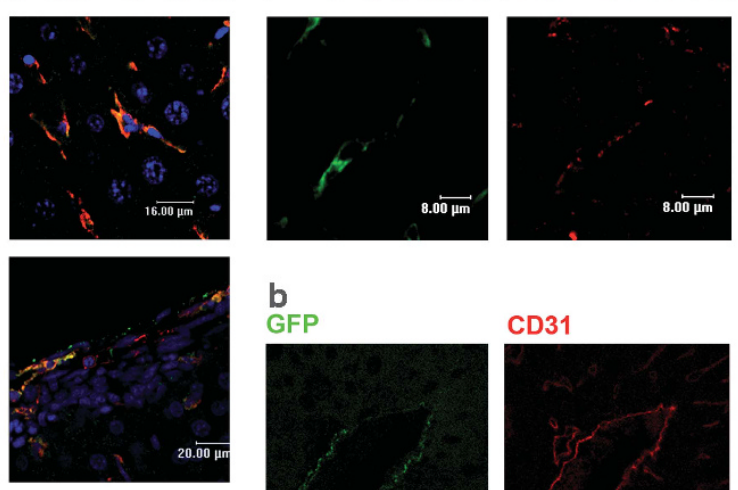

GFP

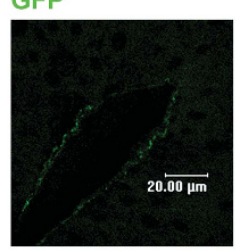

CD31
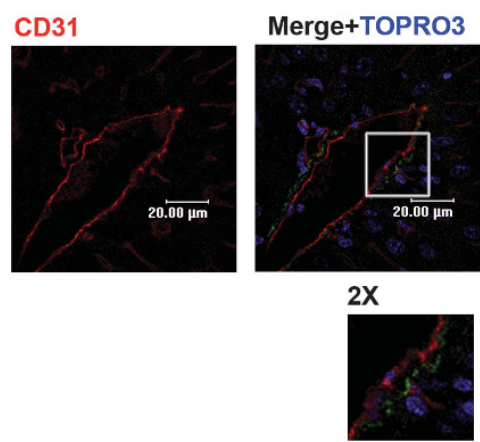

Figure 4 RLSC non-parenchymal derivatives in orthotopic transplants. Immunohistochemical analysis of RLSC-engrafted livers. The RLSC EGFP progeny was detected in untreated CD1 null mice injected at day 1 after birth and killed after 2 months. EGFP non-parenchymal cells expressing GFAP and Desmin were found both (a) scattered in the parenchyma and (b) in subendothelial positions. The endothelium was decorated with CD31 antibody

\section{Discussion}

Taking advance from the RLSC model, we formally proof that single-cell progeny may undergo into two mutually exclusive differentiations having as ending point liver-specific mesenchymal or epithelial cells (Figure 6, yellow box). Are RLSCs mimicking events occurring in vivo? Do RLSCs exist in vivo as stable cell type? While we are not in the position to provide univocal answer to these questions (see Figure 6, red box, for conceivable hypothesis), we believe this is not detrimental to the significance of our data. In fact, despite the limit imposed by a model based on the utilization of cell lines that could in theory have acquired 'non-physiological' properties, this approach seems unique in circumventing a major limit of interpretation of results gathered by means of 'physiological' cellular population (e.g. FACS sorted primary cells): whatever marker utilized for the identification/isolation of precursor cells may be, in theory, shared by distinct cellular subpopulations having distinct properties.

The major conceptual advancement coming from this contribution is due to an unexpected finding: RLSCs, injected in neonatal healthy growing liver, contribute to the formation of the adult organ giving derivatives matching anatomical, morphological and molecular features of both epithelial and mesenchymal cells. In the mesenchymal progeny characterization, both immuno-phenotype and localization of RLSC derivatives are highly indicative of HSCs. The interesting evidences that adult liver may instruct a precursor toward differentiation in HSC should be considered as a self-standing advance as, as yet, HSC precursors in adult animals have to be identified.

In recent years, diverse converging evidence based on in vitro and in vivo experiments utilizing primary or stabilized liver cells allowed for the identification and characterization of bipotential precursors able to give rise to hepatocytes and cholangiocytes. ${ }^{6}$ These precursor cells (i.e. hepatoblasts in normal liver development and oval cells in injured adult organ), as far as we know, give exclusively an epithelial progeny. Several authors have also suggested the possibility that HCS themselves can differentiate into hepatocytes; ${ }^{18-21}$ recent works have demonstrated this in culture ${ }^{22}$ and in vivo with a fate-mapping approach, ${ }^{23}$ thus suggesting that quiescent HSC could be a component of the progenitor compartment in adult liver.

The RLSC clonal progeny was found to belong to two distinct populations that stably maintain their phenotypes in each of the three environments tested. With respect to the in vitro differentiation experiments, RLSCs, previously shown in mitogenic media to differentiate coherently into hepatocytes, were found here, when cultured in low serum, to be also able of accomplishing an alternative mesenchymal differentiation path. The tracking of the initial stages of differentiation highlighted as RLSCs spontaneously endeavor a mutually exclusive path that correlate with the loss of stem signature 


\section{a}

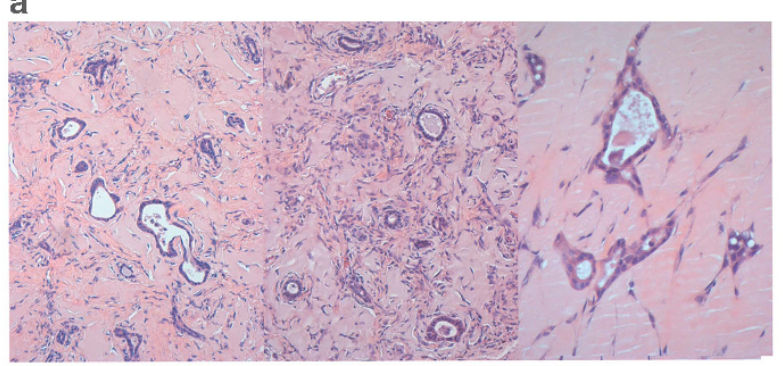

\section{b Ephitelial cells}

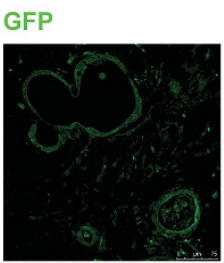

GFP

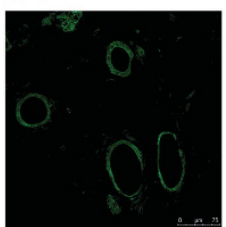

GFP

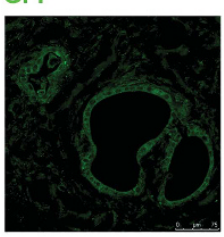

GFP

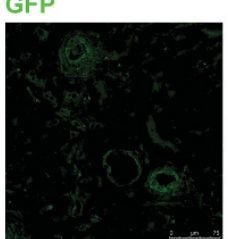

GFP

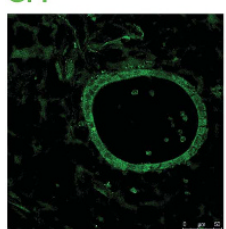

EpCam

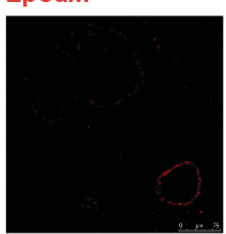

$\alpha$ FP

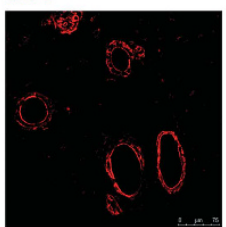

E-Cad

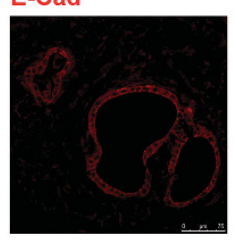

Albumin

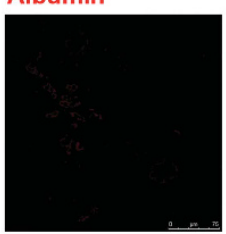

Ck7

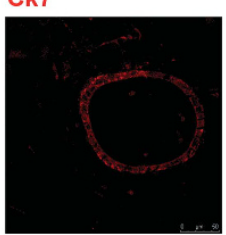

Merge

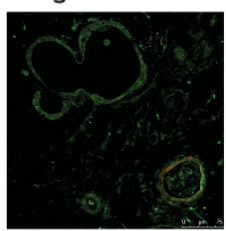

Merge

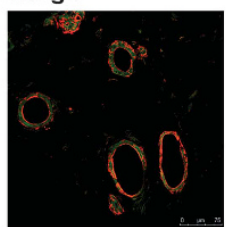

Merge

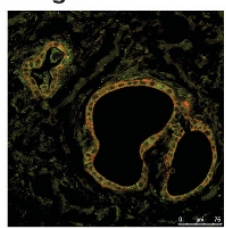

Merge

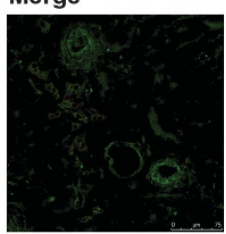

Merge

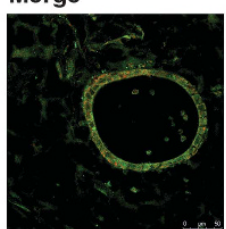

Mesenchymal cells

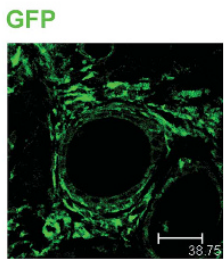

GFP

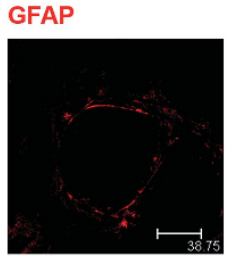

Merge+TOPRO3

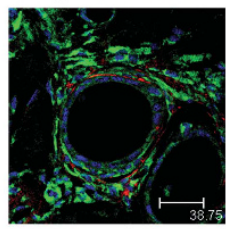

GFAP
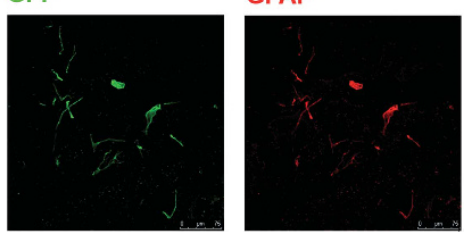

Merge

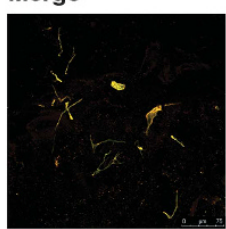

GFP

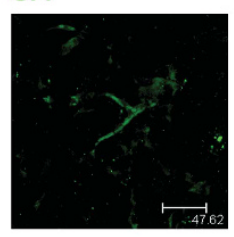

$\alpha$ SMA

Merge+TOPRO3
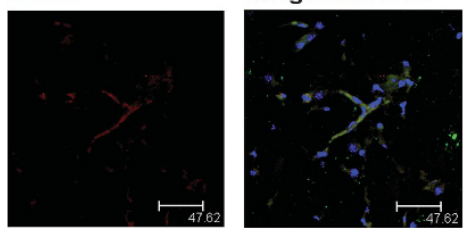

GFAP
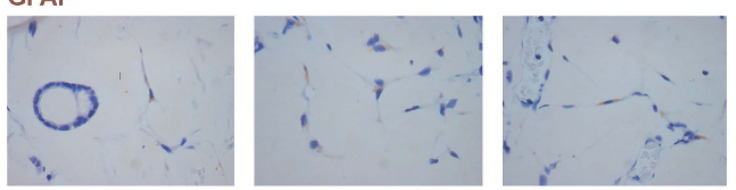

Figure 5 RLSC derivatives in heterotopic transplants. H\&E, Immunohistochemical and Immunofluorescence analysis of RLSCs engrafted in SCID adult null mice. RLSCs were injected in Matrigel in the epifascial region and animals were analyzed 1 month after injection. (a) H\&E revealed epithelial-like RLSC derivatives found either arranged in compact islands or delimiting empty spaces. Mesenchymal-like RLSC derivatives were found scattered in the matrigel scaffold. (b) The EGFP epithelial cells were found to express the hepatic progenitor/hepatocyte markers, EpCAM, $\alpha$ FP, E-Cad, Albumin and CK7. (c) The EGFP mesenchymal cells were found to express GFAP and $\alpha$ SMA by IF and IHC (bottom panel)

(i.e. SCA1). The segregation of lineage-specific markers (i.e. E-cadherin and Vimentin) in subpopulations arising from a bipotent RLSC was strengthened by observations at a single-cell level by means of live imaging. The copresence of distinct cellular subpopulations, bearing specific morphology and observed in the same culture condition, led us to exclude the hypothesis of a mesenchymal to epithelial or epithelial to mesenchymal transitions as the driving force of our observations. Further, dedicated studies may shed light on the molecular mechanisms influencing self-renewal and/or mutually exclusive differentiation choices.

With respect to the results obtained from orthotopic transplantation of RLSCs, notably, both epithelial and mesenchymal derivatives acquire anatomical localization, 

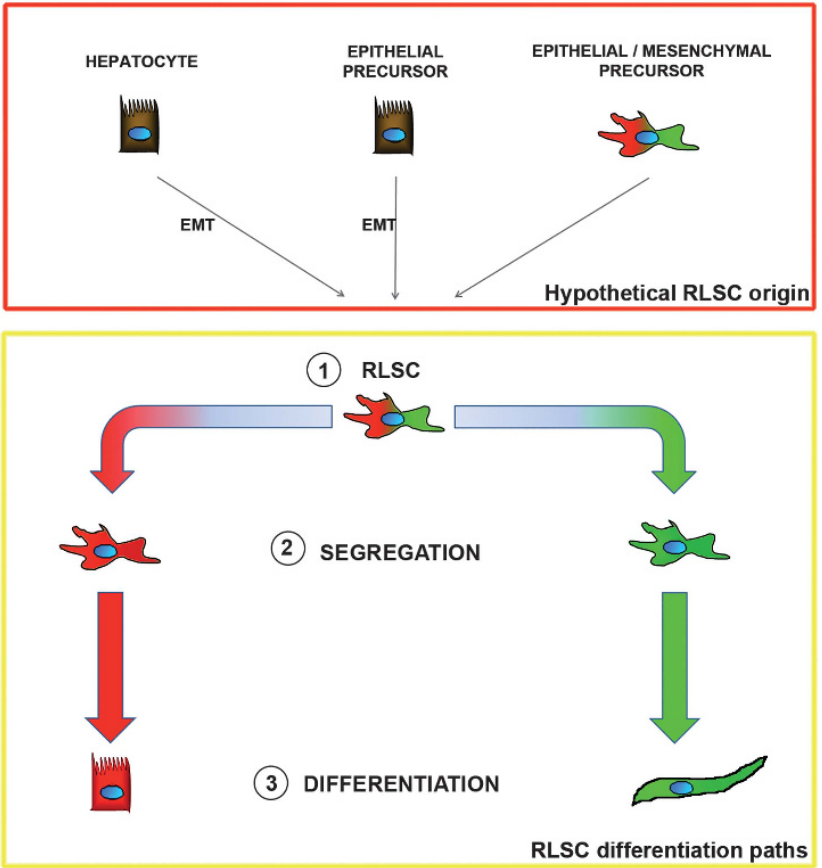

Figure 6 Schematic representation of hypothetical RLSC origin and of observed RLSC differentiation capacity. RLSC have been obtained from singlecell cloning of liver explants. Red box: cells hypothetically giving rise to RLSCs may include epithelial cells that underwent to EMT during the immortalization procedure, thus indicating a broad reprogramming potential of parenchymal cells. Alternatively, an epithelial/mesenchymal RLSC precursor counterpart may exist in vivo. Yellow box: RLSCs were found in cell culture, in heterotopic and in orthotopic transplantations, to undergo into two mutually exclusive differentiations having as ending point mesenchymal or epithelial cells

morphology and immuno-phenotype of liver-specific histotypes. We observed that RLSC progeny colonized non-preconditioned livers of healthy animals (i.e. in competition with the endogenous compartments) with different colonization efficiency in relation to different histotypes. In fact, a limited number of hepatocytes, with respect to a high and unexpected number of mesenchymal/non-parenchymal cells, was found. It is conceivable to hypothesize that, in a growing organ, the stem/precursor compartment contributes mainly to the expansion of the mesenchymal cells, the epithelial components being mainly sustained by the proliferation of differentiated hepatocytes/cholangiocytes.

In heterotopic transplants, again, both RLSC mesenchymal and epithelial derivatives were found: mesenchymal cells expressed markers shared by HSCs (i.e. GFAP, $\alpha$ SMA); epithelial derivatives were found mainly aggregated in tubular structures and expressing markers of endodermal precursors (i.e. EpCAM, $\alpha \mathrm{FP}$ ), and only few cells weakly positive for markers of hepatocytes (i.e. Albumin). This indicates that in vivo only liver-specific cues allow driving a full liver-specific differentiation.

Overall, RLSCs can conceivably be proposed as cells retaining features of the 'meso-endodermal' liver progenitor, whose existence was suggested firstly in chick by Kirby et al., ${ }^{24}$ and then in mouse by Tremblay and Zaret. ${ }^{25}$

Moreover, we believe that our results evoke those previously described in three elegant reports, ${ }^{26-28}$ in which liver progenitor cells bearing an epithelial-mesenchymal habit were showed. However, in the characterization of all of those cells the evidence of a mesenchymal differentiation in vivo is lacking. Moreover, because the cells described there are not of clonal origin, a contamination with mesenchymal cells of the starting cell population was not ruled out. As said, to the best of our knowledge, this is the first time that a liver progenitor cell is described in vivo to differentiate toward a lineage with characteristics attributable to HSCs. On the other hand a limitation of our approach, as yet, is due to the use of fetal/ newborn-derived cells. Only the formal proof that an RLSC homolog compartment do exist in vivo and do contribute to adult liver cells homeostasis will clarify whether RLSC may contribute to the onset of liver fibrosis and whether the precursor compartment should be considered an additional therapeutic target.

\section{Materials and Methods}

Cell culture and manipulation. RLSC lines ${ }^{15}$ were grown in Dulbecco's Modified Eagle's Medium (DMEM) containing 10\% FBS (Lonza Group Ltd, Basel, Switzerland), $2 \mathrm{mmol} / \mathrm{L}$ L-glutamine, $100 \mathrm{mg} / \mathrm{ml}$ penicillin and $100 \mathrm{mg} / \mathrm{ml}$ streptomycin (Gibco, Grand Island, NY, USA) on collagen I (Transduction Laboratories, Lexington, UK) coated dishes (Falcon-BD, Franklin Lakes, NJ, USA). RLSCs were genetically marked by infection with the recombinant PINCO retroviral vector, which encodes the enhanced EGFP in accordance with Grignani et al. ${ }^{29}$ and subcloned by limiting dilution procedure (at a 0,2 cell/well dilution).

Animal care and treatment. Animal care and experiments were carried out according to the criteria outlined in the 'Guide for the Care and Use of Laboratory Animals' of the National Academy of Sciences, published by the National Institutes of Health (NIH publication 86-23 revised 1985).

In vivo orthotopic transplantation. $C D 1$-nude newborn mice (1 day after birth) were transplanted by transcutaneous injection into the liver with $1 \times 10^{6}$ EGFP + RLSCs in $15 \mathrm{ml}$ phosphate-buffered saline (PBS) using a 30-gauge needle (Hamilton Bonaduz AG, Bonaduz, Switzerland). Mice were killed by cervical dislocation after 30 to 60 days. The livers were removed and dissected; the specimens were observed under a fluorescent microscope and the colonized regions were formalin fixed, mounted onto OCT and cooled by liquid nitrogen.

In vivo heterotopic transplantation in growth factors reduced matrigel. EGFP + RLSCs $\left(1 \times 10^{6}\right)$ were suspended in $1 \mathrm{ml}$ of growth factors reduced Matrigel (BD Biosciences Labware, San Diego, CA, USA) with an equal quantity of HUVEC (Cambrex Corporation, Walkersville, NJ, USA). Aliquots of about $0,7 \mathrm{ml}$ of suspension were injected subcutaneously into the backs of SCID beige mice (Charles River Laboratories, Raleigh, NC, USA), carefully positioning the needle between the epidermis and the muscle layer. Mice were killed by cervical dislocation after 20 days and transplants were immediately fixed for histology.

Endothelial cells were grown in Clonetics EGM-2 BulletKit (Cambrex Corporation, Walkersville, NJ, USA) following the manufacturer's instructions.

Immunofluorescence. Formalin fixed frozen liver tissues were sliced $(7 \mu \mathrm{M})$ and stained with: anti-GFP Ab 1/200 (Santa Cruz Biotechnology, Santa Cruz, CA USA, sc-8334), anti-EGFP Ab 1/600 (Abnova, Taipei City, Taiwan, MAB1765), anti-HNF4 $\alpha$ Ab 1/100 (Abcam, Cambridge, UK, ab41898), anti-Albumin Ab 1/200 (Novus Biologicals, Cambridge, UK, ab19196), anti-PanCytokeratin Ab 1/500 (Dako, Glostrup, Denmark, z0622), anti-Cytokeratin 7 Ab 1/200 (Abcam, Cambridge, UK, ab9021), anti-Desmin Ab 1/200 (Thermo Scientific, Fremont, CA, USA, RB-9014), anti-GFAP Ab 1/200 (Millipore, Billerica, MA, USA, MAB3402), anti-CD31 Ab 1/200 (BD Biosciences, Franklin Lakes, NJ, USA, 55027), anti-EpCAM Ab 1/200 (BD Biosciences 552370), anti- $\alpha$ FetoProtein Ab 1/200 (Santa Cruz Biotechnology sc-8108), anti-E-Cadherin Ab 1/50 (BD Biosciences 610181) and anti- $\alpha \mathrm{SMA} A b$ 1/200 (Sigma-Aldrich, St Louis, MO, USA, A5228).

Secondary antibodies (Alexa-Fluor 488 and Alexa-Fluor 594 diluted 1/500) were from Molecular Probes, Eugene, OR, USA. The nuclei were costained with 
TOPRO3 (Invitrogen, San Diego, CA, USA). Preparations were examined with a Leica TCS2 confocal microscope.

Immunolocalization was performed using standard immunoperoxidase (DAB reaction) and counterstained with hematoxylin.

Five micrometers-thick heterotopic transplant sections, embedded in paraffin, were stained with hematoxylin and eosin. For immunocytochemistry, cells were grown on collagen I-coated dishes and fixed and treated as previously described. ${ }^{15}$

Flow cytometry. Cells were suspended in $\mathrm{Ca}^{++} \mathrm{Mg}^{++}$-free PBS containing $20 \% \mathrm{FCS}$, mouse $\mathrm{lgG}(40 \mu \mathrm{g} / \mathrm{ml})$, incubated for $10 \mathrm{~min}$ on ice and labeled with fluorochrome-conjugated $\mathrm{mAbs}$ for $30 \mathrm{~min}$ on ice. For intracellular staining, cells were treated with $10 \%$ saponin in PBS. Cell fluorescence was analysed with the FACS Calibur (Becton Dickinson, Franklin Lakes, NJ, USA).

Antibodies: FITC-conjugated anti-SCA1 (BD Biosciences); Alexa-Fluor 647 Mouse anti-Nestin (BD Biosciences); pan Cytokeratin antibody (C-11) (Phycoerythrin) (ab52460 Abcam); anti-E-Cadherin (CD324) Per-CP-eFluor 710 (e-Bioscence, San Diego, CA, USA); anti-NCadherin C-terminus clone EPR1792Y (Millipore) and anti-Vimentin Ab (2707-1 Epitomics, Burlingame, CA, USA) both followed by secondary Alexa-Fluor 596 .

Time-lapse video microscopy for clonal assay. A mixed population of RLSCs and EGFP-expressing RLSCs (ratio of 20:1) were seeded on a collagen-coated micro-dish Grid-500 (Ibidi, Martinsried, Germany, 81166) and mounted on a micro-incubator stage (OKO Lab, Italy) at $37^{\circ} \mathrm{C}$ and $5 \% \mathrm{CO}_{2}$ for time-lapse analysis. Time-lapse video microscopy was performed using a Nikon $\mathrm{Ti}$ Eclipse microscope equipped with a $\times 10$ PlanFluor objective starting from a single GFP positive cell per field. Phase contrast images were acquired every $5 \mathrm{~min}$ and fluorescent GFP signals were acquired every $60 \mathrm{~min}$ with a specific filters set. After 5 days, the grid positions of different clones were annotated and processed for immunostaining. Cells were fixed with PFA $4 \%$ for $10 \mathrm{~min}$ and Triton-X100 $0,1 \%$ for $5 \mathrm{~min}$ and incubated with different antibodies. Primary antibodies were: Mouse anti-E-Cadherin (BD Biosciences) and Rabbit antiVimentin (Epitomics). Secondary antibodies were: anti-rabbit AlexaFluor 488 (Invitrogen) and anti-mouse AMCA (Jackson Immunoresearch, Newmarket, Suffolk, UK). Immunofluorescence analysis was carried out under an Olympus Vanox microscope equipped with a $\times 40$ objective and a SPOT CCD camera (Diagnostic Instruments, Sterling Heights, MI, USA).

\section{Conflict of Interest}

The authors declare no conflict of interest.

Acknowledgements. This work was supported by the Italian Cancer Research Foundation, Ministry of Health, The Ministry of University and Scientific Research and the European Molecular Biology Organization. We especially thank Patrizia Lavia and Giulia Guarguaglini (CNR, Rome) for their help in live imaging experiments.

\section{Author contributions}

$A C$ and LA: conception and design, collection and/or assembly of data, data analysis and interpretation. AC, BS, CC, VC, CM, FG, MDP and TK: collection and/ or assembly of data. MR and DB supply of reagents/materials/analysis tools and editing of the manuscript. PB: conception and design. LA and MT: manuscript writing. MT: conception and design, financial support, data analysis and interpretation

1. Dellavalle A, Sampaolesi M, Tonlorenzi R, Tagliafico E, Sacchetti B, Perani $L$ et al. Pericytes of human skeletal muscle are myogenic precursors distinct from satellite cells. Nat Cell Biol 2007; 9: 255-267.

2. Tanaka EM, Ferretti $P$. Considering the evolution of regeneration in the central nervous system. Nat Rev Neurosci 2009; 10: 713-723.
3. Li L, Clevers H. Coexistence of quiescent and active adult stem cells in mammals. Science 2010; 327: 542-545.

4. Nagy P, Bisgaard HC, Thorgeirsson SS. Expression of hepatic transcription factors during liver development and oval cell differentiation. J Cell Biol 1994; 126: 223-233.

5. Sell S. Liver stem cells. Mod Pathol 1994; 7: 105-112.

6. Duncan AW, Dorrell C, Grompe M. Stem cells and liver regeneration. Gastroenterology 2009; 137: 466-481.

7. Alison MR, Golding M, Sarraf CE, Edwards RJ, Lalani EN. Liver damage in the rat induces hepatocyte stem cells from biliary epithelial cells. Gastroenterology 1996; 110: $1182-1190$

8. Theise ND, Saxena R, Portmann BC, Thung SN, Yee H, Chiriboga L et al. The canals of Hering and hepatic stem cells in humans. Hepatology 1999; 30: 1425-1433.

9. Asahina K, Zhou B, Pu WT, Tsukamoto H. Septum transversum-derived mesothelium gives rise to hepatic stellate cells and perivascular mesenchymal cells in developing mouse liver. Hepatology 2011; 53: 983-995.

10. Geerts A. History, heterogeneity, developmental biology, and functions of quiescent hepatic stellate cells. Semin Liver Dis 2001; 21: 311-335.

11. Sato M, Suzuki S, Senoo H. Hepatic stellate cells: unique characteristics in cell biology and phenotype. Cell Struct Funct 2003; 28: 105-112.

12. Friedman SL. Hepatic stellate cells: protean, multifunctional, and enigmatic cells of the liver. Physiol Rev 2008; 88: 125-172.

13. Winau F, Hegasy G, Weiskirchen R, Weber S, Cassan C, Sieling PA et al. Ito cells are liverresident antigen-presenting cells for activating $T$ cell responses. Immunity 2007; 26: 117-129.

14. Choi SS, Diehl AM. Epithelial-to-mesenchymal transitions in the liver. Hepatology 2009; 50: 2007-2013.

15. Conigliaro A, Colletti M, Cicchini C, Guerra MT, Manfredini R, Zini R et al. Isolation and characterization of a murine resident liver stem cell. Cell Death Differ 2008; 15: 123-133.

16. Colletti M, Cicchini C, Conigliaro A, Santangelo L, Alonzi T, Pasquini E et al. Convergence of Wnt signaling on the HNF4alpha-driven transcription in controlling liver zonation. Gastroenterology 2009; 137: 660-672.

17. Bissig KD, Le TT, Woods NB, Verma IM. Repopulation of adult and neonatal mice with human hepatocytes: a chimeric animal model. Proc Natl Acad Sci USA 2007; 104: 20507-20511

18. Roskams T. Different types of liver progenitor cells and their niches. J Hepatol 2006; 45: $1-4$.

19. Yin L, Lynch D, Sell S. Participation of different cell types in the restitutive response of the rat liver to periportal injury induced by allyl alcohol. J Hepatol 1999; 31: 497-507.

20. Schmitt-Graeff A, Jing R, Nitschke R, Desmouliere A, Skalli O. Synemin expression is widespread in liver fibrosis and is induced in proliferating and malignant biliary epithelial cells. Hum Pathol 2006; 37: 1200-1210.

21. Koenig S, Probst I, Becker H, Krause P. Zonal hierarchy of differentiation markers and nestin expression during oval cell mediated rat liver regeneration. Histochem Cell Biol 2006; 126: 723-734.

22. Kordes C, Sawitza I, Müller-Marbach A, Ale-Agha N, Keitel V, Klonowski-Stumpe H et al. CD133 + hepatic stellate cells are progenitor cells. Biochem Biophys Res Commun 2007; 352: 410-417.

23. Yang L, Jung Y, Omenetti A, Witek RP, Choi S, Vandongen HM et al. Fate-mapping evidence that hepatic stellate cells are epithelial progenitors in adult mouse livers. Stem Cells 2008; 26: 2104-2113.

24. Kirby ML, Lawson A, Stadt HA, Kumiski DH, Wallis KT, McCraney E et al. Hensen's node gives rise to the ventral midline of the foregut: implications for organizing head and heart development. Dev Biol 2003; 253: 175-188.

25. Tremblay KD, Zaret KS. Distinct populations of endoderm cells converge to generate the embryonic liver bud and ventral foregut tissues. Dev Biol 2005; 280: 87-99.

26. Dan YY, Riehle KJ, Lazaro C, Teoh N, Haque J, Campbell JS et al. Isolation of multipotent progenitor cells from human fetal liver capable of differentiating into liver and mesenchymal lineages. Proc Natl Acad Sci USA 2006; 103: 9912-9917.

27. Yovchev MI, Grozdanov PN, Zhou H, Racherla H, Guha C, Dabeva MD. Identification of adult hepatic progenitor cells capable of repopulating injured rat liver. Hepatology 2008; 47: 636-647.

28. Inada M, Follenzi A, Cheng K, Surana M, Joseph B, Benten D et al. Phenotype reversion in fetal human liver epithelial cells identifies the role of an intermediate meso-endodermal stage before hepatic maturation. J Cell Sci 2008; 121: 1002-1013.

29. Grignani F, Kinsella T, Mencarelli A, Valtieri M, Riganelli D, Grignani F et al. High-efficiency gene transfer and selection of human hematopoietic progenitor cells with a hybrid EBV/retroviral vector expressing the green fluorescence protein. Cancer Res 1998; 58 : 14-19. 